\title{
Extracting health-related causality from twitter messages using natural language processing
}

\author{
Son Doan*, Elly W. Yang, Sameer S. Tilak, Peter W. Li, Daniel S. Zisook and Manabu Torii \\ From The Sixth IEEE International Conference on Healthcare Informatics (ICHI 2018) \\ New York, NY, USA. 4-7 June 2018
}

\begin{abstract}
Background: Twitter messages (tweets) contain various types of topics in our daily life, which include health-related topics. Analysis of health-related tweets would help us understand health conditions and concerns encountered in our daily lives. In this paper we evaluate an approach to extracting causalities from tweets using natural language processing (NLP) techniques.

Methods: Lexico-syntactic patterns based on dependency parser outputs are used for causality extraction. We focused on three health-related topics: "stress", "insomnia", and "headache." A large dataset consisting of 24 million tweets are used.
\end{abstract}

Results: The results show the proposed approach achieved an average precision between 74.59 to $92.27 \%$ in comparisons with human annotations.

Conclusions: Manual analysis on extracted causalities in tweets reveals interesting findings about expressions on healthrelated topic posted by Twitter users.

Keywords: Twitter, Causality, Causal relationships, Cause-effect, Natural language processing (NLP)

\section{Background}

Twitter messages (tweets) have been a unique public resource for monitoring health-related information, including, but not limited to, disease outbreaks [1-3], suicidal ideation $[4,5]$, obesity $[6]$, and sleep issues $[7,8]$. Tweets provide diverse types of information about Twitter users, such as users' behaviors, lifestyles, thoughts, and experiences. Automated causality extraction from tweets can help gather unique health-related information complementary to that from other data sources, such as research literature and electronic medical records. Despite the importance, the topic has not been extensively studied yet. This exploratory study considered automated extraction of attributable causes of health problems and concerns. We investigated whether causes of a given health

\footnotetext{
* Correspondence: Son.Doan@kp.org

Medical Informatics, Kaiser Permanente Southern California, San Diego, CA 92130, USA
}

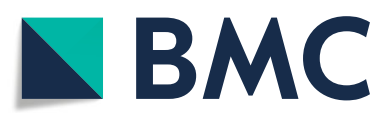

(c) The Author(s). 2019 Open Access This article is distributed under the terms of the Creative Commons Attribution 4.0 International License (http://creativecommons.org/licenses/by/4.0/), which permits unrestricted use, distribution, and reproduction in any medium, provided you give appropriate credit to the original author(s) and the source, provide a link to the Creative Commons license, and indicate if changes were made. The Creative Commons Public Domain Dedication waiver (http://creativecommons.org/publicdomain/zero/1.0/) applies to the data made available in this article, unless otherwise stated. problem or concern can be extracted from Twitter messages using natural language processing (NLP) techniques. We specifically focused on three health-related topics: stress, insomnia, and headache.

Text mining from tweets poses various challenges [9-11]. One of the challenges in studying causal relations is to accurately identify a small fraction of relevant tweets from a large data collection. In addition, language constructs within tweets are often informal and can make identification of causal relationships difficult. In this study, therefore, we aimed at precise extraction of causal relationships that are explicitly stated within a sentence. For example, given a tweet "Excessive over thinking leads to insomnia", our goal is to extract "excessive over thinking" as a cause of "insomnia." To this end, we created a set of lexico-syntactic patterns to extract "cause" information for a given "effect". Quantitative and qualitative analyses were performed on causal relationships extracted from 24 million tweets. 
Cause-effect relation extraction has been actively studied in the NLP field. There are two main approaches used for the task: 1) pattern/rule-based methods and 2) machine learning-based methods [12-15]. For example, Khoo et al. applied a dependency parser to each sentence and searched for extraction patterns in an obtained dependency graph using graph pattern matching. They reported an accuracy of $68.1-76.3 \%$ in identifying causality mentions in the Medline abstracts [16]. Girju and Moldovan used lexico-syntactic patterns involving causation verbs to extract cause-effect relations using pattern matching. They reported an accuracy of $65.6 \%$ on a news article corpus [12]. Similarly, Cole et al. [15] used a syntactic parser to identify triples of subject, verb, and object and applied various rules to determine which of these triples represent causal relations. They reported a precision and recall of 94.44 and $61.82 \%$, respectively, on a news article corpus. Ittoo and Bouma automatically extracted causal patterns from Wikipedia and reported a precision of $76.5 \%$ and a recall of $82.0 \%$ on domain-specific documents (customer complaint and engineers' repair action on medical equipment) $[17,18]$. Recently, machine learning approaches have also been used to tackle causality extraction tasks [19-21]. Gijru used decision trees (C4.5) trained on 6000 sentences to extract causal relations and reported a precision of $73.91 \%$ and recall of $88.69 \%$ on a test set of 1200 sentences in the newswire domain [22]. Similarly, Blanco et al. [23] used decision trees to classify whether or not a pattern correctly encodes a causation and reported an average F-score of $89.5 \%$ on 1068 instances (75\% training and 25\% test) on a general English text corpus. Other works used support vector machines (SVMs) and conditional random fields (CRFs) with lexical, syntactic and semantic features and reported F-scores ranging from 0.82 to 0.85 on general English text corpus [24, 25]. Although the performance measures reported for machine learning-based methods are high compared with the pattern/rule-based approach, model training requires a large amount of manually-annotated data and a new model needs to be trained when target domains are changed. A comprehensive survey on causal relation extraction in the general NLP domain can be found in Asghar et al. [13].

Social media in general and Twitter in particular have been found as a useful and impactful resource in healthrelated surveillance studies. Twitter data have been used to mine topics related to depression [26, 27], mental health [28], stress and relaxation [29], and tobacco use [30]. Most common techniques for Twitter mining in the health-related domains are keyword/dictionary look-up and machine learning. Among the machine learning algorithms applicable to the tasks, support vector machines (SVM), logistic regression, and neural networks have been commonly used [1,3,29-31]. Although there have been Twitter studies in the health domain that concern causal relationships, such as the study of adverse reactions caused by drugs $[32,33]$ or various factors causing stress and relaxation [29], their focus is on a specific application and they do not investigate causal relation extraction itself. We believe there is a lack of studies on causality extraction from tweet in the health domain. To our best knowledge, this is the first study focusing on this problem.

\section{Methods \\ Dataset}

We used a corpus of 24 million tweets, collected from four cities (New York, Los Angeles, San Francisco and San Diego) over 4-month period (Sep 30, 2013 and Feb 10, 2014). Twitter Streaming API was used to retrieve $1 \%$ of all the tweets from these cities during the time period. This corpus was previously used to study stress and relaxation tweets [29]. Three terms: stress, insomnia, and headache, were selected as the target "effects".

\section{NLP pipeline}

We aimed to develop a general method to extract causalities that is readily applicable to a new extraction target. We adopted an NLP framework that can leverage syntactic information to extract causal relations using a pattern/rule-based method. The NLP pipeline for this task is shown in Fig. 1. First, the corpus was filtered using the target keywords. Next, a series of basic NLP components were applied: sentence splitter, lemmatizer, Part-of-Speech (POS) tagger, and a dependency parser. Finally, causal relations were identified based on syntactic relations generated by the dependency parser. We used the CoreNLP package [34] (release version 3.8), a widely used Java library providing various NLP functionalities. The default settings and pre-trained models in the package were used for sentence splitter, lemmatizer, and POS tagger. For the parser, we selected the Probabilistic Context-Free Grammar (PCFG) parser and the pre-trained English model in the package, which generates a constituent tree for an input sentence and then converts it into a dependency graph. A dependency graph consists of vertices representing tokens (words and punctuations) and edges representing dependency relations among tokens [35]. Dependency relations are convenient for the purpose of extracting term relations in a sentence. Specifically, we used "Universal Dependencies" [35], which has a general annotation scheme to support multi-lingual and has been widely used in NLP community [35, 36]. Among the several options provided for dependency graph generation in the CoreNLP package, the method generateEnhancedDependencies that produces enhanced dependencies was used to derive dependency graphs from parsed trees. 


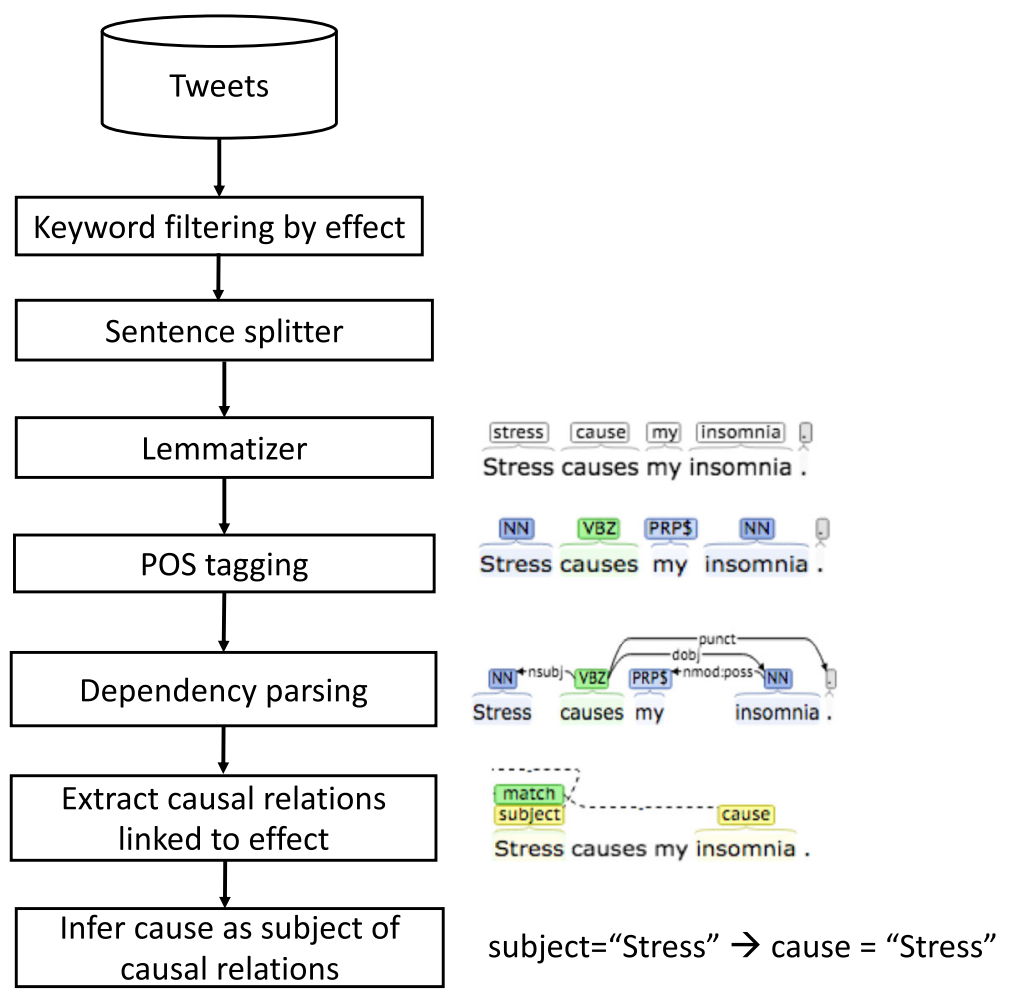

Fig. 1 A general framework for causality extraction from Twitter messages

\section{Cause-effect relation extraction}

There are many different ways to state cause and effect relations, including verb phrases and noun phrases. As a result, we created rule set templates including trigger verbs and nouns. For example, a tweet containing "A caused B" has "caused" as a trigger verb and similarly "A result in B" has "result (in)" as a trigger verb. We initially created a list of trigger verbs and nouns by searching synonyms of "cause", "result" and "reason" in WordNet (version 3.1), a widely used lexical database [37]. Ambiguous synonyms, such as "do" and "get", were removed from the list. In the end, we selected seven verbs: "cause", "stimulate", "make", "derive", "trigger", "result", and "lead." Similarly, we selected three nouns: "result", "reason", and "cause."

The cause-effect relations were determined based on trigger terms as below:

Trigger verb (active): "A <trigger> B", where "<trigger>" is one of the seven selected verbs, e.g., "Stress caused insomnia".

Trigger verb (phrasal, active): "A <trigger > <preposition> B", where <trigger > is "cause", "result", or "reason" and < preposition > is "in", "to", or "from". E.g., "Stress results in insomnia".

Trigger verb (passive): "A < be> <trigger> by B", where $<$ be $>$ is an appropriate form of be-verb, such as "is", and
$<$ trigger $>$ is a past participle form of a trigger verb. E.g. "Stress was caused by insomnia".

Trigger nouns: "A <be> <trigger> of B", where <trigger $>$ is "cause", "result", or "reason" with an appropriate determiner. E.g., "Insomnia is a result of stress".

Based on this principle, we created a set of six patterns to identify cause-effect relationship. To identify these patterns in a dependency graph derived from a sentence, we used CoreNLP Semgrex [38], which facilitates subgraph pattern matching over a dependency graph. The details of rules and their examples are listed in Table 1. For example, Rule 1 in Table 1 " \{\}$=$ subj <subj (\{word: /cause $/\}=$ target $>\operatorname{dobj}\{\}=$ cause)" indicates that the rule will match a sentence, such as "Stress caused my insomnia", where "Stress" is matched with the pattern " \{\}$=$ subj" and "insomnia" is matched with the pattern " \{\}$=$ cause." (Fig. 1). The base form of the trigger verbs and nouns could be used in these rules as the words were lemmatized by the Lemmatizer component of the CoreNLP library; for example, the base form "cause" is used in the rule to represent "causes", "caused" and "causing".

The final step was to extract causalities from identified cause-effect relations. We extracted the triple <cause, trigger, effect>, where effect is one of the three health-related topics of our focus: insomnia, stress, and headache. 
Table 1 Rule set to extract causal relations FROM TWEETS

\begin{tabular}{|c|c|c|c|}
\hline \# & Causal relation types & Dependency rules & Examples \\
\hline 1 & A (noun) caused B & \{\}$=$ subj $<$ subj $(\{+$ Causal verb +$\}=$ target $>$ dobj \{\}$=$ cause $)$ & Stress causes insomnia \\
\hline 2 & A (verb-ing) caused B & \{\}$=$ subj $<$ csubj $(\{+$ Causal verb +$\}=$ target $>$ dobj \{\}$=$ cause $)$ & $\begin{array}{l}\text { Over thinking can increase anxiety and } \\
\text { cause insomnia. }\end{array}$ \\
\hline 3 & B was caused by $A$ & \{\}$=$ ncsubjpass $<$ nsubjpass $(\{+$ Causal verb +$\}=$ target $>/$ nmod:agent $/\{\}=$ cause $)$ & My insomnia was caused by stress. \\
\hline 4 & $A$ is a reason of $B$ & Causal noun $+<$ nsubj $(\{\}=$ target $>/$ nmod:of $/\}=$ cause $)$ & Stress is a reason of my insomnia \\
\hline 5 & $\begin{array}{l}\text { B was caused by A } \\
\text { (verb-ing) }\end{array}$ & \{\}$=$ nsubj $<$ nsubjpass $(\{\}=$ target $>/$ advcl:by $/+$ Causal noun $)$ & Insomnia was caused by overthinking \\
\hline 6 & A results "in/to/from" B & Causal verb $+<[$ nc] subj $(\{\}=$ target $>/$ nmod:(to|in|from $) /\{\}=$ cause $)$ & Stress results to insomnia. \\
\hline
\end{tabular}

\section{Results}

We observed that the number of tweets containing specific health-related cause-effect relationships is small despite the large size of the Twitter corpus used. Specifically, the number of matched sentences was 501 out of 29,705 for stress (1.6\%), 72 out of 3827 for insomnia $(1.8 \%)$, and 94 out of 11,252 for headache $(0.8 \%)$. The final causalities extracted were 41 for insomnia, 98 for stress, and 42 for headache. The details of the matching rules and the number of extracted causalities are shown in Table 2.

Table 2 also indicates that the majority of the relations were extracted by the pattern "A cause B" (Rule 1), followed by the pattern 'A result in/to/from B' (Rule 6). The rest of rules were used much less. This may suggest that Twitter users generally prefer direct and concise expressions. Notably, similar or the same phrases are repeated in collected tweets. For example, similar phrases "missing someone causes insomnia", "missing someone often causes insomnia", and "missing someone causes insomnia like symptoms" were found.

\section{Quantitative analysis}

As stated, we aimed at precise extraction of causal relation using syntactic information. Therefore, we used the precision to evaluate our proposed method in this study, i.e., we manually reviewed tweets that were selected by

Table 2 Results when applying rule set in table i to a corpus of 24 millions tweets. The last rows indicates the numbers of tweets extracted with given effects (INSOMNIA, STRESS AND HEADACHE)

\begin{tabular}{llll}
\hline Matched rule \# & $\begin{array}{l}\text { Insomnia } \\
\text { (of 3827) }\end{array}$ & $\begin{array}{l}\text { Stress } \\
\text { (of 29,705) }\end{array}$ & $\begin{array}{l}\text { Headache } \\
\text { (of 11,252) }\end{array}$ \\
\hline 1 & 58 & 381 & 78 \\
2 & 4 & 12 & 3 \\
3 & 0 & 4 & 1 \\
4 & 1 & 21 & 2 \\
5 & 0 & 32 & 0 \\
6 & 9 & 51 & 10 \\
Total & 72 & 501 & 94 \\
\# extracted causalities & 41 & 98 & 42 \\
\hline
\end{tabular}

the proposed method, instead of reviewing the entire Twitter corpus. The precision is calculated as the number of positive instances annotated by human annotators divided by the number of tweets the system found. The micro-average precision was calculated as the sum of all positives instances across all three categories divided by the sum of tweets the system found. Three human annotators [SD, EY, MT] discussed the annotation criteria and manually annotated the system outputs. We considered two annotation criteria: strict annotation and relaxed annotation. With the strict annotation, extracted relations were considered correct only when the cause of the target effect is clearly and explicitly stated. In the relaxed annotation, negated or hypothetical statements were additionally considered as correct extraction. For example, "Cell phone radiation can cause insomnia", where the statement is hypothetical, was annotated as a false positive case in strict annotation, but a true positive case in relaxation. The disagreement in annotation were resolved by discussions among the annotators.

Table 3 shows the precision when comparing system outputs to human annotations. It shows that the microaverage for strict and relaxation is 74.59 and $92.27 \%$, respectively. It also indicates that finding causal relationships for "headache" is more difficult than "insomnia" and "stress". The large variations between the strict and relaxation evaluation $(74.59 \%$ vs. $92.27 \%)$ also indicates that hypothetical assertions and negation play important roles in determining causal relationships in Twitter messages.

Table 3 Precision of extracted causalities when cOMPARING TO HUMAN ANNOTATORS

\begin{tabular}{lll}
\hline & Strict evaluation & $\begin{array}{l}\text { Relax evaluation } \\
\text { (exclude hypothetical } \\
\text { assertions and negation) }\end{array}$ \\
\hline Insomnia & $73.81 \%$ & $88.10 \%$ \\
Stress & $82.65 \%$ & $96.94 \%$ \\
Headache & $56.10 \%$ & $85.37 \%$ \\
Micro-average & $74.59 \%$ & $92.27 \%$ \\
\hline
\end{tabular}




\section{Qualitative analysis}

We further manually analyzed the causes of insomnia, stress and headache extracted by the system. Below are several findings.

\section{Insomnia}

We found that the most frequent cause related to insomnia was "missing someone". Other causes include overthinking, social media (Facebook, Twitter), and hunger. Below are some examples of tweets and matching rules extracted from this topic:

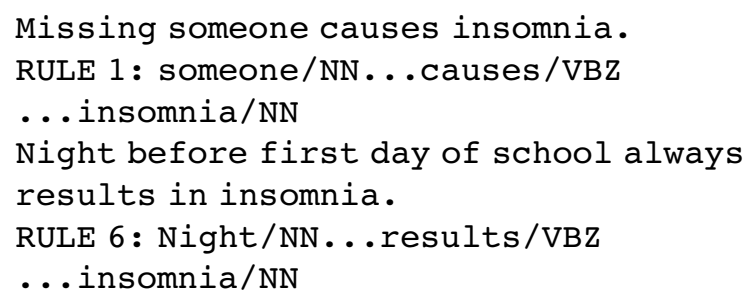

\section{Stress}

Frequent topics related to stress for Twitter users include school, money, emails, computer games, and physical pains. Below are some of examples and matching rules for this topic.

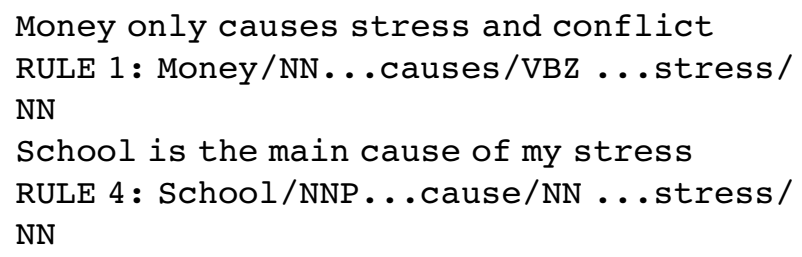

\section{Headache}

We observed the causes of headache reported in tweets include people, stress, crying, and listening. Below are some examples:

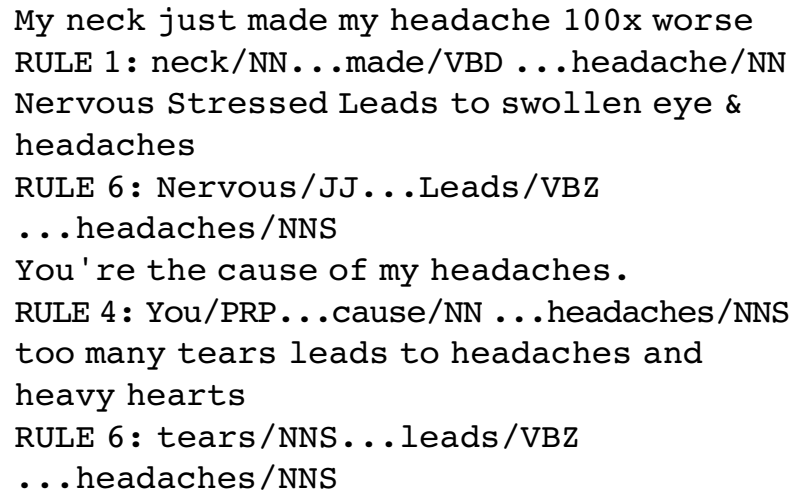

Error analysis showed that false positive errors are often caused by complex sentences, such as "Keeping to myself makes life way more stress free", or interrogative sentences, such as "Could this be the cause of my insomnia?". Some false positive cases were due to sentences that do not explicitly indicate causality, such as "I wouldn't say it causes insomnia though". In such sentences, there are dependency links between subjects and given effects, and they match a rule in the extraction rule set. Including extraction rules that are more restricted and conducting more semantic analysis after extraction should further improve the precision.

\section{Discussion}

Identifying target tweets precisely and efficiently is a primary concern in mining Twitter messages, which contains very large data and time-sensitive information. The goal in our experiment was to correctly identify tweets referring to causal information in a large data set using NLP. A dependency parser and associated NLP techniques were used to help improve precise information extraction. We also manually reviewed tweets identified by the proposed approach. We observed that the number of extracted causal relations is small. However, evaluation showed that it achieved a high precision. This indicates that using lexicon-syntactic relations derived from dependency parser yields high precision, which is an important factor in mining information from a large data set.

We further applied the framework to in-house clinical text since the proposed approach is general and flexible to customize. With modifications in our existing rules, we can correctly detect, within the clinical text, the association of a disease or symptoms with the patient. For example, the rule " \{\}$=$ subj $<$ nsubj (\{lemma:/have $/\}=$ target $>/$ dobj/ \{\}$=$ cause)" can correctly assign assertions of disease not associated to patient such as "Parkinson's" disease in the sentence "Patient says his roommate has Parkinson's and is concerned about his diagnosis" or the second mention of "asthma" in "Patient has no history of asthma, son has severe asthma". We believe it can benefit an NLP engine and has potential applications in the future.

\section{Limitations}

The study has several limitations. First, the number of our rules and patterns are currently small and they may miss some expressions reporting cause-effect relations in sentences. Second, in this study we only considered a simple case of causal relation explicitly reported within one sentence. In reality, there can be cause-effect relation reported across sentences. Third, in Twitter messages, there are diverse ways in expressing cause-effect relations, including hashtags. The current approach that relies on syntactic patterns is unable to extract such information. Fourth, the data we used in this study is a 
small fraction (1\%) from the real-world Twitter data. Although it was found that the fraction of tweets reporting causal relations was small, scalability of the method and the workflow is of important consideration in practice. Finally, we did not consider synonymous expressions of target concepts in the current study. It was beyond the scope of our exploratory study, but it must of great significance, especially when the synonymous expressions are not limited to noun phrases. e.g., "can't sleep" as a synonymous expression of "insomnia."

\section{Conclusions}

In this paper, we presented an NLP approach to extracting causality from Twitter messages. The results on four months Twitter data revealed some interesting findings about different health-related topics. In the future we will focus more on semantic analysis, such as hashtags as well as multi-sentence causality extractions from tweets.

\section{Abbreviations}

CRF: Conditional Random Field; NLP: Natural Language Processing; POS: Partof-Speech; SVM: Support Vector Machine

\section{Acknowledgements}

We thank Twitter Inc. for providing API functions to access the Twitter data and members in the KPSC Medical Informatics group for their helpful suggestions and comments on the manuscript. We also thank anonymous reviewers for useful and constructive comments to improve the manuscript.

\section{Funding}

Publication costs are funded by Kaiser Permanente.

\section{Availability of data and materials}

Not applicable.

\section{About this supplement}

This article has been published as part of BMC Medical Informatics and Decision Making Volume 19 Supplement 3, 2019: Selected articles from the first International Workshop on Health Natural Language Processing (HealthNLP 2018). The full contents of the supplement are available online at https:// bmcmedinformdecismak.biomedcentral.com/articles/supplements/volume19-supplement-3.

\section{Authors' contributions}

Conceived and proposed the research idea: SD. Designed and implemented the experiments: SD, MT. Analyzed the data: MT, EWY, SD. Wrote the first draft of the manuscript: SD. Contributed to the writing of the manuscript: SD, EWY, SST, PWL, DSZ, MT. Agreed with manuscript results and conclusions: SD, EWY, SST, PWL, DSZ, MT. Jointly developed the structure and arguments for the paper: MT, EWY, SST, PWL, DSZ, SD. Made critical revisions and approved the final version: MT, EWY, SST, PWL, DSZ, SD. All the authors reviewed and approved the final manuscript.

\section{Ethics approval and consent to participate}

This research is not human research and did not require IRB approval.

\section{Consent for publication}

Not applicable.

\section{Competing interests}

The authors declare that they have no competing interests.

\section{Publisher's Note}

Springer Nature remains neutral with regard to jurisdictional claims in published maps and institutional affiliations.

Published: 4 April 2019

\section{References}

1. Paul MJ, Dredze M, Broniatowski D. Twitter improves influenza forecasting. PLOS Currents Outbreaks. Edition 1. 2014;1-13.

2. Stefanidis A, Vraga E, Lamprianidis G, Radzikowski J, Delamater PL, Jacobsen $\mathrm{KH}$, et al. Zika in twitter: temporal variations of locations, actors, and concepts. JMIR public Heal Surveill. 2017;3:e22.

3. Collier N, Doan S. Syndromic classification of Twitter messages. Electron Healthc. Springer Berlin Heidelberg; 2012. p. 186-95.

4. Sueki $H$. The association of suicide-related twitter use with suicidal behaviour: a cross-sectional study of young internet users in Japan. J Affect Disord. 2015;170:155-60.

5. Burnap P, Colombo G, Amery R, Hodorog A, Scourfield J. Multi-class machine classification of suicide-related communication on twitter. Online Soc networks media. 2017;2:32-44.

6. So J, Prestin A, Lee L, Wang Y, Yen J, Chou W-YS. What do people like to "share" about obesity? A content analysis of frequent retweets about obesity on twitter. Health Commun. 2016;31:193-206.

7. Mclver DJ, Hawkins JB, Chunara R, Chatterjee AK, Bhandari A, Fitzgerald TP, et al. Characterizing sleep issues using twitter. J Med Internet Res. 2015;17:e140.

8. Jamison-Powell S, Linehan C, Daley L, Garbett A, Lawson S. "I can't get no sleep": discussing \#insomnia on twitter. Proc 2012 ACM Annu Conf hum factors Comput Syst - CHI '12. New York, New York: ACM Press; 2012. p. 1501.

9. Collier N, Nguyen ST, Nguyen MTN. OMG U got flu? Analysis of shared health messages for bio-surveillance. Proc 4th Symp Semant Min Biomed. Cambridge, UK; 2010. p. 18-26.

10. Culotta A. Towards detecting influenza epidemics by analyzing Twitter messages. Proc First Workshop on Social Media Analytics. New York: ACM Press; 2010;115-22.

11. Mowery D, Smith H, Cheney T, Stoddard G, Coppersmith G, Bryan C, et al. Understanding depressive symptoms and psychosocial stressors on twitter: a Corpus-based study. J Med Internet Res. 2017;19:e48.

12. Girju R, Moldovan D. Text mining for causal relations. Proc FLAIRS Conf. 2002:360-4.

13. Asghar N. Automatic extraction of causal relations from natural language texts: a comprehensive survey; 2016.

14. Girju R, Moldovan D, Blanco E, Castell N, Moldovan D, Luo Z, et al. Causal relation extraction. Proc FLAIRS Conf. 2006;2016:421-30.

15. Cole SV, Royal MD, Valtorta MG, Huhns MN, Bowles JB. A lightweight tool for automatically extracting causal relationships from text. Conf Proc - IEEE SOUTHEASTCON. 2006;2006:125-9.

16. Khoo CSG, Chan S, Niu Y. Extracting causal knowledge from a medical database using graphical patterns. Proc 38th Annu Meet Assoc Comput Linguist - ACL '00. Morristown, NJ: Association for Computational Linguistics; 2000. p. 336-43.

17. Ittoo A, Bouma G. Extracting Explicit and Implicit Causal Relations from Sparse, Domain-specific Texts. Proc 16th Int Conf Nat Lang Process Inf Syst. Berlin, Heidelberg: Springer-Verlag; 2011. p. 52-63.

18. Ittoo A, Bouma G. Minimally-supervised learning of domain-specific causal relations using an open-domain corpus as knowledge base. Data Knowl Eng. 2013;88:142-63.

19. Gordon AS, Kozareva Z, Roemmele M. SemEval-2012 Task 7: Choice of Plausible Alternatives: An Evaluation of Commonsense Causal Reasoning. SemEval 2012. 2012;394-8.

20. Yang $X$, Mao K. Multi level causal relation identification using extended features. Expert Syst Appl. 2014;41:7171-81.

21. Rink B, Bejan CA, Harabagiu S. Learning textual graph patterns to detect causal event relations. Artif Intell. 2010:265-70.

22. Girju R. Automatic detection of causal relations for Question Answering. Proc ACL 2003 Work Multiling Summ Quest answering. Morristown, NJ: Association for Computational Linguistics; 2003. p. 76-83.

23. Blanco E, Castell N, Moldovan D. Causal Relation Extraction. Proc 6th Int Conf Lang Resour Eval Lr 2008. 2008;310-3.

24. Girju R, Nakov P, Nastase V, Szpakowicz S, Turney P, Yuret D. Classification of semantic relations between nominals. Lang Resour Eval. 2009;43:105-21. 
25. Pakray P, Gelbukh A. An open-domain cause-effect relation detection from paired nominals. In: Gelbukh A, Espinoza FC, Galicia-Haro SN, editors. Nature-inspired Comput Mach learn. Cham: Springer International Publishing; 2014. p. 263-71.

26. De Choudhury M, Counts S, Horvitz E. Social media as a measurement tool of depression in populations. Proc 5th Annu ACM Web Sci Conf - WebSci '13. New York, New York, USA: ACM Press; 2013. p. 47-56.

27. Lachmar EM, Wittenborn AK, Bogen KW, McCauley HL.

\#MyDepressionLooksLike: Examining Public Discourse About Depression on Twitter. JMIR Ment Heal. 2017;4:e43.

28. Reece AG, Reagan AJ, Lix KLM, Dodds PS, Danforth CM, Langer EJ. Forecasting the onset and course of mental illness with twitter data. Sci Rep. 2017;7:13006

29. Doan S, Ritchart A, Perry N, Chaparro JD, Conway M. How do you \#relax when You're \#stressed? A Content Analysis and Infodemiology Study of Stress-Related Tweets. JMIR public Heal Surveill. 2017;3:e35.

30. Myslín M, Zhu SH, Chapman W, Conway M. Using twitter to examine smoking behavior and perceptions of emerging tobacco products. J Med Internet Res. 2013;15:e174.

31. Chen T, Dredze M. Vaccine images on twitter: analysis of what images are shared. J Med Internet Res. 2018;20(4):e130.

32. $\operatorname{Cocos}$ A, Fiks AG, Masino AJ. Deep learning for pharmacovigilance: recurrent neural network architectures for labeling adverse drug reactions in twitter posts. J Am Med Inform Assoc. 2017:24:813-21.

33. O'Connor K, Pimpalkhute P, Nikfarjam A, Ginn R, Smith KL, Gonzalez G. Pharmacovigilance on twitter? Mining tweets for adverse drug reactions. AMIA Annu Symp Proc. 2014;2014:924-33.

34. Manning C, Surdeanu M, Bauer J, Finkel J, Bethard S, McClosky D. The Stanford CoreNLP Natural Language Processing Toolkit. Proc 52nd Annu Meet Assoc Comput Linguist Syst Demonstr. Stroudsburg, PA: Association for Computational Linguistics; 2014. p. 55-60.

35. Nivre J, Marneffe M De, Ginter F, Goldberg Y, Manning CD, Mcdonald R, et al. Universal Dependencies v1: A Multilingual Treebank Collection. Proc 10th Int Conf Lang Resour Eval (LREC 2016). 2016;1659-66.

36. Mcdonald R, Nivre J, Quirmbach-brundage Y, Goldberg Y, Das D, Ganchev K, et al. Universal Dependency Annotation for Multilingual Parsing. Proc 51st Annu Meet Assoc Comput Linguist. 2013:92-7.

37. Miller GA. WordNet: a lexical database for English. Commun ACM. 1995;38:39-41.

38. Chambers N, Cer D, Grenager T, Hall D, Kiddon C, et al. Learning alignments and leveraging natural logic. Proc Workshop on Textual Entailment and Paraphrasing. 2007:165-70

Ready to submit your research? Choose BMC and benefit from:

- fast, convenient online submission

- thorough peer review by experienced researchers in your field

- rapid publication on acceptance

- support for research data, including large and complex data types

- gold Open Access which fosters wider collaboration and increased citations

- maximum visibility for your research: over $100 \mathrm{M}$ website views per year

At BMC, research is always in progress.

Learn more biomedcentral.com/submissions 